\title{
Regulation of Hemocytes in Drosophila Requires dappled Cytochrome b5
}

\author{
Kurt Kleinhesselink • Corinna Conway • \\ David Sholer • Irvin Huang • Deborah A. Kimbrell
}

Received: 23 September 2010/ Accepted: 20 December 2010/Published online: 30 January 2011

(C) The Author(s) 2011. This article is published with open access at Springerlink.com

\begin{abstract}
A major category of mutant hematopoietic phenotypes in Drosophila is melanotic tumors or nodules, which consist of abnormal and overproliferated blood cells, similar to granulomas. Our analyses of the melanotic mutant dappled have revealed a novel type of gene involved in blood cell regulation. The dappled gene is an essential gene that encodes cytochrome b5, a conserved hemoprotein that participates in electron transfer in multiple biochemical reactions and pathways. Viable mutations of dappled cause melanotic nodules and hemocyte misregulation during both hematopoietic waves of development. The sexes are similarly affected, but hemocyte number is different in females and males of both mutants and wild type. Additionally, initial tests show that curcumin enhances the dappled melanotic phenotype and establish screening of endogenous and xenobiotic compounds as a route for analysis of cytochrome b5 function. Overall, dappled provides a tractable genetic model for cytochrome b5, which has been difficult to study in higher organisms.
\end{abstract}

Keywords Hemocytes - dappled C Cytochrome b5 - Melanotic tumors · Granulomas

GenBank Accession Number HQ864711 dappled.

K. Kleinhesselink · C. Conway · D. Sholer · I. Huang · D. A. Kimbrell ( $₫)$ Department of Molecular and Cellular Biology, University of California,

1 Shields Avenue, Davis, CA 95616, USA

e-mail: dakimbrell@ucdavis.edu

Present Address:

K. Kleinhesselink

Seminis Corporation, Woodland, CA, USA

Present Address:

D. Sholer

Genentech Inc., Vacaville, CA, USA 


\section{Introduction}

Drosophila is well studied as a model of innate immunity in humans and has been instrumental in establishing principles of defense and gene signaling pathways that involve both immune induction and hematopoiesis (Lemaitre and Hoffmann 2007). The Drosophila Toll pathway mediates the response to microbial infections, and humans were subsequently found to have a family of Toll receptors that also respond to infections. Other shared immune signaling pathways are the Imd (immune deficiency)/TNF1 (tumor necrosis factor 1), JNK (Jun kinase), and JAKSTAT (Janus kinase-STAT transcription factor) pathways. Activation of NFkB transcription factors, which regulate target genes such as cytokines and antimicrobial proteins, is a key shared feature (Wang and Ligoxygakis 2006). Like the signaling pathways, the cell and tissue components of the Drosophila and human immune systems are analogous (Kimbrell and Beutler 2001). In Drosophila, the hemocytes and the fat body mediate, respectively, the major cellular and humoral arms of the response. Hemocytes participate in immune signaling to the fat body, phagocytosis, damaged tissue surveillance, encapsulation of foreign intruders, and remodeling of cells during development and also respond to aberrant self-cells to form melanotic tumors/pseudotumors (Babcock et al. 2008; Brennan et al. 2007; Gateff 1994; Rizki and Rizki 1983; Tepass et al. 1994; Vass and Nappi 2000). The fat body, like the mammalian liver, produces abundant humoral response molecules, including antimicrobial peptides released into the hemolymph (Aggrawal and Silverman 2007).

Drosophila produces three types of hemocytes: plasmatocytes, lamellocytes, and crystal cells (Crozatier and Meister 2007). Plasmatocytes account for $~ 95 \%$ of total blood cells and most functions. In particular, plasmatocytes are responsible for the phagocytosis of microorganisms during an infection and apoptotic cells during development. Lamellocytes are produced only in response to parasites or by mutations that produce melanotic tumors. Crystal cells constitute $\sim 5 \%$ of total blood cells and are involved in melanization. As in vertebrates, Drosophila blood cells also have two developmental origins: one early in the embryonic head mesoderm and another during larval development in the lymph gland (Evans et al. 2003). Molecular cascades and factors regulating hematopoiesis are also shared with humans and involve many pathways, for example, Toll, JAK/STAT, Hedgehog, Notch, GATA, and Runx (Evans and Banerjee 2003; Mandal et al. 2007).

A major category of mutant hematopoietic phenotypes in Drosophila is that of melanotic tumors or nodules. Melanotic nodules may be in the hemocoel or associated with the lymph glands and consist of abnormal and overproliferated blood cells that are covered by lamellocytes and melanized, similar to the immune response to a parasitic wasp egg (Luo et al. 1995; Meister and Lagueux 2003; Minakhina and Steward 2006b; Stofanko et al. 2008). Mutations in a variety of genes and genetic pathways have been found to produce this phenotype, such as JAK-STAT, which is also associated with blood cell overproliferation in humans, the Toll pathway, and Ras/MAPK (Asha et al. 2003; Li 2008; Minakhina and Steward 2006a; Zettervall et al. 2004). Some other genes with this mutant phenotype are $\operatorname{air}^{8}$, encoding ribosomal protein s6 (Watson et al. 1992); black pearl, 
encoding a DnaJ homolog (Becker et al. 2001); l(3)malignant blood neoplasm, encoding a protein with human cytokeratin homology (Gateff 1994); yantar, encoding a conserved arginine-rich protein (Sinenko et al. 2004); and the Polycomb group gene multi sex combs (Remillieux-Leschelle et al. 2002). The melanotic tumor genes are an important category to identify and define in order to understand blood cell function and regulation.

In a P-element enhancer detector screen concentrating on immune-related tissues and phenotypes, we identified the melanotic tumor gene dappled ( $d p l d$ ) (Rodriguez et al. 1996). Enhancer detector expression of dappled was high in the fat body, oenocytes, ring gland, and gut. Lethal and viable mutations of dappled produced melanotic tumors, and lethal mutation was also associated with aberrant tissue morphology, for example, of the fat body and gut (Rodriguez et al. 1996). A distinguishing feature of dappled mutants is consistent melanotic tumor formation, in contrast to other melanotic tumor mutants, which typically have low and variable rates. We now report identification of dappled as a member of the cytochrome b5 family. Cytochrome b5 is a conserved, ubiquitous, small hemoprotein that participates in electron transfer in multiple biochemical reactions and pathways, such as fatty acid desaturation, cytochrome P450-catalyzed reactions, sterol metabolism, and conversion of methemoglobin (Schenkman and Jansson 2003). Cytochrome b5 is thus involved in processes such as metabolism of endogenous and xenobiotic compounds, maintenance of oxygen levels, and production of steroid hormones (Locuson et al. 2007; Miller 2005; Yamazaki and Shimada 2006). Cytochrome b5 genetics in higher organisms has not been very amenable to study, and dappled provides a new avenue of approach for study of cytochrome b5 functions. In this report, we characterize the dappled mutant hemocyte phenotype and present the potential to study cytochrome b5 function by screening for compounds that affect the dappled hemocyte phenotype.

\section{Materials and Methods}

\section{Drosophila Stocks and Rearing}

Oregon $\mathrm{R}$ flies were used as the standard wild-type strain. The dappled mutants are the $d p l d^{M L B}(97 / 16)$ and $d p l d^{E J L}(142 / 2)$ strains of Rodriguez et al. (1996). Experiments were performed using $d p l d^{M L B} / d p l d^{E J L}$ transheterozygotes or $d p l d$ mutants hemizygous with $D f(2 R) S T 1$ (Bloomington Stock Center). The hemeseGal4 strain is that of Zettervall et al. (2004). The UAS-dappled transgenic strain was made using the cDNA clone RE66521 from the Bloomington Drosophila Genome Project, which matched our splice product for dappled mRNA (see below). The insert of plasmid RE66521 was isolated using the EcoRI and BamHI restriction enzyme sites, ligated into the EcoRI and $X b a \mathrm{I}$ sites of the pUAST vector using $B a m \mathrm{HI} / X b a \mathrm{I}$ linkers, and transgenic strains made by Genetic Services, Inc. Insertion strains of UAS-dappled into chromosomes X, 2, and 3 were recovered. All stocks were raised on the Bloomington Stock Center food recipe in a $25^{\circ} \mathrm{C}$ incubator. For the curcumin-raised flies, the food, before cooling, was supplemented with 
Fig. 1 The dappled locus. a Four P-element insertions in dappled/CG2140 produce mutations with a melanotic tumor phenotype (k09716, k00107, k14202, and 01857). These four dappled insertions and the 03427 and 05643 insertions are included within the k08815 deletion. Insertions 03610 and 08492 are outside the dappled and k08815 regions. One dappled mRNA transcript has been detected, and it has one intron spanning from +120 to +1712 (dotted line). The numbers 55, 56, and 57 indicate the positions of PCR primers used to test for alternative mRNA splicing products; only the 55-57 pair produced a PCR product. Black bars indicate the dappled protein coding region. b Sequence of the dappled/CG2140 gene. The sequence of the PCR fragment obtained from primers 55 and 57 to total adult RNA was combined with the sequence data for CG2140 at Flybase (Tweedie et al. 2009). Transcribed sequence with $5^{\prime}$ UTR and $3^{\prime}$ UTR is shown in capital letters. The protein encoding sequence is underlined, with the ATG start site shaded gray, and the translated amino acid sequence is below the underline. Bold and italicized sequences indicate the primers 55, 56, and 57

$20 \mathrm{mg} / \mathrm{ml}$ curcumin (Sigma), and parents were placed onto the food for mating and egg laying. Compare with Gupta et al. (2009), in which doses for mice were 5, 30, and $60 \mathrm{mg} / \mathrm{kg}$ orally, daily for 19 days, and note that at $100 \mathrm{mg} / \mathrm{ml}$, flies had high lethality in addition to developmental delay.

\section{Molecular Genetic Analysis}

Genomic DNA fragments flanking the dappled 97/16 (k09716) and 142/2 (k14202) P-element insertion sites were recovered by the plasmid rescue technique (Wilson et al. 1989), sequenced (Davis Sequencing), and aligned to the genome (Blast). The PCR primers used to compare the 88/15 (k08815) strain P-element insertion sequences AQ034113 and AQ025871 (Tweedie et al. 2009) to wild-type sequences were TATCGTCGCTGTGCTTACAGAT (60, forward) and CTAAACCTCCAA TTCGGCTATG (61, reverse) at the AQ034113 end and CTCCACGCGTAATA TACCCATT (58, forward) and AGTCATGGATGCGTAGTCACAC (59, reverse) at the AQ025871 end. In order to confirm the 88/15 insertion site, PCR fragments that resulted from the P-element end primer RsyII (Tweedie et al. 2009) in combination with primers 59 and 60 were sequenced. The PCR primers used to test for possible alternative splicing of CG2140/dappled were sequences 55, 56, and 57 (Fig. 1). The sequence of the single transcript detected was translated to an amino acid sequence and placed into the cytochrome b5 family by Blast comparison. Clustal $\mathrm{W}$ was used to align the dappled cytochrome b5 sequence to selected other cytochrome b5 proteins.

\section{Northern Blot Analysis}

Total RNA was isolated using the Totally RNA kit (Ambion), and poly-A RNA was isolated using the Oligotex Direct mRNA Purification kit (Qiagen). The RNA was run on formaldehyde gels and transferred overnight using Ambion Northern Max reagents. The dappled probe was 642 bp defined by the primers 55 and 57 (Fig. 1). This 642 bp PCR fragment was subcloned using the TA cloning kit (Invitrogen), following the manufacturer's protocol. After blue/white screening to select the colony, PCR and sequencing were used to confirm the insert and determine orientation. Transcription used T7 polymerase and ${ }^{32} \mathrm{P}$-labeled UTP and the Ambion Strip EZ kit following the manufacturer's protocol. The rp49 probe was made using 
(a)

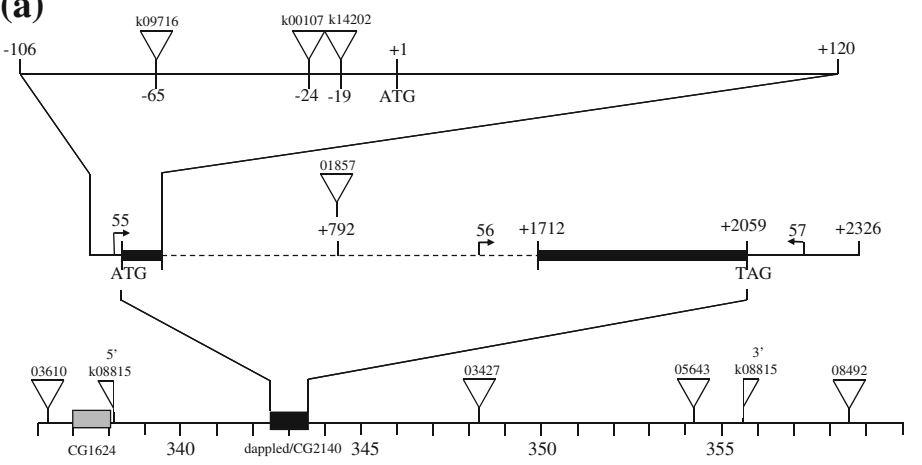

(b)

- 140 agccatcttgctgcgcgccagtgtgaccgccggccaccAGTTCTGTGAGACCGTGCGCCC

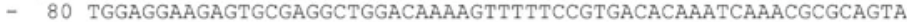

- 20 GAAATCAACAAGTGATCACCATGTCGAGCGAGGAAACAAAGACTTTCACGCGGGCCGAGG

+40 TCGCCAAGCACAACACGAACAAGGACACCTGGCTGCTCATACACAACAACATCTACGACG $\begin{array}{llllllllllllllllllll}A & K & H & N & T & N & K & D & T & W & \text { L } & \text { L } & \text { I } & \text { H } & \text { N } & \text { N } & \text { I } & \text { Y } & \text { D } & \text { V }\end{array}$

+100 TCACCGCCTTCCTCAACGAGGTGAGAATCGCACGGAACACGTCGCAGCTGAGTGCCACCG $\begin{array}{llllllllll}\text { T } & \text { A } & \text { F } & \text { L } & \text { N } & \text { E }\end{array}$

+160 CCCCGCACCCGCTCCCACCACTAAATGGGGGTCATCGTTTGCTATTGGGCCTTATCTGGG

+220 TTTCACTTGTCCCGATGAGCTAATGGGCCCAGCCCCAGCACAAGCCCAAGCCCACGTAGC

+280 CACATGTAGCGACTCTAACTTGCATCGCGGGGCAATTAGGTCTGTACTTTGACCCCCGGA

+340 CGGTAGTTTGACCTGCGTCCGGATTTCGCAGATTTGCTGCGACGTCGAAACTTCCAGCAG

+400 CCACTTTCGCCCAGCTGTCGAAAGAGCTCCACATGGCGAAACTATCCATTTGCATACATG

+460 TATATATGTGTGTGATGGGCGACGAGgGTCAAGTGTTAAGCGAAATCCGCGTCTGCATTT

+ 520 GCATCTCATTTGATTAACGGACTCCGATTTGAATTCGGATTCTTATGCAAGTGCACCACC

+580 GACAAACCGACTGCCGCCGGCTCCGAAAAGTGTATCACCCTGACGCAGCAGTAGGGTCAT

+640 CACCAAATCTATACATATAGACTACACAAGCATTCCCGCTATCTGCGgGATCTAAGATAA

+700 GCTCTGTGAAAATTCCGATCTAACGATGACGTTGCACTTTAGGTACACGCTGAAAATAAC

+760 AATAGTGAGAGTGACCAAGCATCTAATTTATAATTCCATTCCAAAGTTTCAGTCCGCCTA

+820 TCGACCGCAGCCATCCCCAGTCCATGTTTAAAACATGCCCCTAATCTGATCCGCTTGGTC

+880 CTCTTCCATCTTAAGGAATAACATTAGCTACTGTTACCTTGCTATCTGCGTAACAGCATC

+940 ACCAGACAAATCGCAAATTAGACTTCCAGATTTTAGAGCGTTTGCTGGATCTAAAGATTA

+1000 TTTTTTACGTGTTTATTCGTTATTTATTCAACATGATCAGTTTATTTTGACGAATCTTAT

+1060 TCAATGTGTTCCTGCATATGTATGTTCCGATTTGTCATATATAAACCAACACAGTAATT

+1120 ACAATATAATCATCGAAATTAGATAAGGCTAAGTTGGACTATGTAAAAGTTTAAAGAATT

+1180 GTGAAAATAATTATGAGATACGCTTATCATCGATTCAATTGCACTCGGCCACCTTGAAGA

+1240 GTTTGGCTAAAATTACTTTAGCTTATCACTCAGCACAATTGAGGCGAAACAACTTGTTTG

+1300 CTTTTAATAGTAATTAATAACAATAATTACATCATACTTGCTCCGCGGCTTTTTCAGCAT

+1360 GACTCTAAGCTATAGTACAAAGTTTCAACATTATTATAATGGTACAATCAAAACGTAACT

+1420 TCGCTCGAGTTCTTAGGATCAAAGTTTCTGTAACTTTAT TGGCTTATAAGTGCCAAATTA

+1480 AATCTGTAACTGAAATTAGTTGTGATATTTTACTTTCAACGCA.ACGGAAGTATA.AAATA

+1540 TTACCATAAAGCTTCCGCATAAACGAATGTTTCGAAGCATTCCCGACTTTATACCTTGAT

+1600 ACCATACCCTCTGCTGTCTGGTCCATGAAATTCAGGGTCAGACGTCCATTAGCATTTATC

+1660 CAAGATCAGCCAACAGTTATTTATGCGAATATATTAATCTGTCTCTTTTCCTCATCCCGG

+1720 TGGCGAAGAGGTGCTCATCGAGCAGGCCGGCAAAGATGCCACGGAGAACTTTGAGGACGT

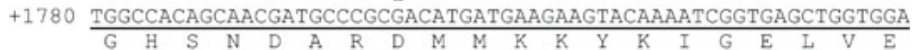

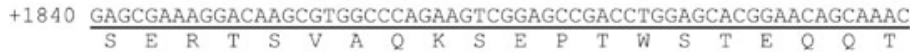

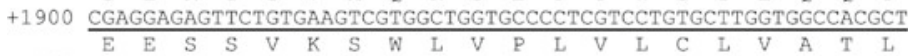

$+1960 \frac{\text { CTTCTACAAGTTCTTCTTTGGCGGTGCCAAGCAGGAGCCACAATGCCCAGCAGAGACTTT }}{\mathrm{F}}$

+2020 CCAGCCCGCTCCGGAGTCGCTTAGTTAATCAACAAACGGAGAGCCCGAGAGTCACATGAG +2080 GAGCGGGACAGTGGAGCGGGAAAGCTTTTTATAGTAAATTTGTGCTAAGTTTTAACGATA

+2140 ATTTTTGGCATTTGGCGTTTTTGTACAACCCTCTACACGTGTAATTCCTTCAGACGGCGG

+2200 TTCTAGACTCTAAAAGTAATTTATCCCATTGATATTCCAACATTTGAGGCACGGA.ACGAA

+2260 AACGATAAGTTTTTATTCGCATGTATGTACATTAAACATATTTCCAAAAGCaagcaaaca 
the primers TCCTTCCAGCTTCAAGATGACC (forward) and AGAAGTTCCTG GTGCACAACGT (reverse). Hybridization was overnight, and a Fuji phosphoimager and ImageJ were used to analyze the hybridization signal.

\section{Blood Cell Counts}

Third-instar larvae were developmentally staged as early versus late wandering phase based on retention in the gut of food dye (Zettervall et al. 2004). The BrightLine Counting Chamber (Fisher) hemocytometer was used to count blood cells stained with monochlorobimane dye (Tirouvanziam et al. 2004) or GFP-expressing blood cells as in Zettervall et al. (2004), except that instead of red food color we used blue (Deep Blue Shade, Esco Foods, Inc.). Siblings that were heterozygous for dappled were distinguished based on either a $\mathrm{CyO}$ balancer with the marker $\mathrm{y}+$ (i.e., $y ;+/ d p l d$ vs. $y ;+/ C y O, y+$ ) or B-galactosidase staining to indicate presence or absence of a dpld enhancer trap (Rodriguez et al. 1996). Separate counts of blood cells in $y$ versus $y+$ larvae established that $y$ did not affect the blood cell counts. Determination of sex was by the size of the gonads or by marking the sexes with $y$ versus $y+$. Hemocyte counts from different samples were compared using the $t$ test, and $P$ values were calculated using VassarStats (http://faculty.vassar.edu/ lowry/VassarStats.html).

\section{Results}

\section{Cytochrome b5, the Encoded Product of dappled}

The original dappled alleles, $d p l d^{E J L}$ and $d p l d^{M L B}$, result from the P-element insertions (P-lacW) in strains 142/2 (k14202) and 97/16 (k09716), respectively (Rodriguez et al. 1996). The $d p l d^{E J L}$ allele is homozygous and hemizygous lethal, and $d p l d^{M L B}$ is homozygous and hemizygous viable. Heteroallelic $d p l d^{E J L} / d p l d^{M L B}$ is also viable and, like $d p l d^{E J L}$ and $d p l d^{M L B}$ homozygotes and hemizygotes, has melanotic tumors (Rodriguez et al. 1996). In order to identify the gene associated with the melanotic tumor phenotype, we molecularly cloned and sequenced the dappled gene. We used the technique of plasmid rescue to isolate the genomic DNA flanking the k14202 and k09716 insertions. Sequencing of the flanking DNA and alignment to the genome placed both insertions into the $5^{\prime}$ UTR of CG2140 (Tweedie et al. 2009), with k09716 46 bp farther upstream than k14202 (Fig. 1).

In contrast to our results, genome annotation assigned dappled as CG1624 based on the position of the lethal P-element insertion 88/15 (k08815), which did not complement the 142/2 insertion (Spradling et al. 1999). We therefore investigated this discrepancy in the identification of dappled. The $5^{\prime}$ and $3^{\prime}$ flanking sequences for the k08815 insertion are $3^{\prime}$-AQ034113 and 5'-AQ025871. Alignment of these flanking sequences on the latest Drosophila genome sequence, however, places them 176,059 bp apart. There are several potential causes for this gap in the sequence: multiple insertions, an error in data reporting, or a large deletion. To test for the accuracy of the k08815 flanking sequence, primers were designed to (1) flank 
the $3^{\prime}$ and $5^{\prime}$ insertion sites of the k08815 transposon and (2) extend from a P-element end primer ( $\mathrm{p}$ RsyII) into a flanking sequence. The resultant PCR fragments supported the published flanking sequences, as wild-type products were not observed in the mutant strain and both insertion sites were verified with the P-element end primer (Fig. 1). Finally, these PCR products were sequenced to provide confirmation of the flanking sequences. These results indicate that k08815 is not a simple insertion but is associated with additional disruption at its genomic position. We set out to differentiate between either multiple insertions or a large deletion by performing complementation tests with other lethal insertion strains in the region. Complementation tests confirmed that a large deletion accompanies the k08815 insertion (Fig. 1). Complementation and mapping also placed two more insertions, k00107 and 01857, into the 5' UTR and intron of CG2140/dappled, respectively (Fig. 1).

We tested these various dappled mutants for alterations in transcription, further confirming that the dappled phenotype results from an effect on CG2140, by performing Northern blot analysis using RNA from dappled mutants. This analysis showed a decrease in CG2140 transcript levels in the dappled mutants compared with the Oregon R controls (Fig. 2). The dappled strains are hypomorphic, with the lethal alleles stronger. The lethal allele $d p l d^{E J L}$ expresses approximately $10 \%$ of wild-type embryonic RNA levels, whereas the viable allele $d p l d^{M L B}$ has $\sim 80 \%$ of wild-type adult RNA levels. Analysis of the transcripts by RT-PCR and sequencing showed one splice variant, with a single intron (Fig. 1).
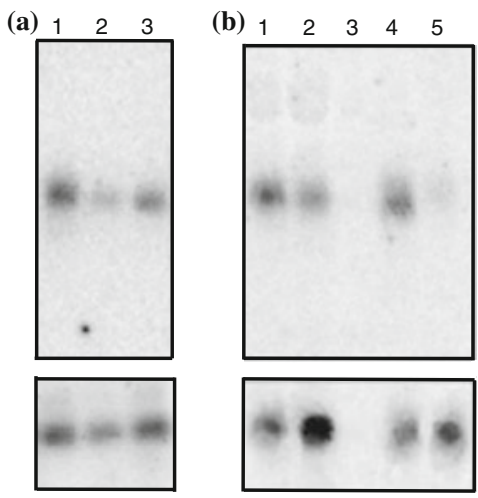

Fig. 2 The dappled transcript levels are reduced in dappled mutants. Northern blot analysis of dappled transcripts shows reduced levels in mutants compared with wild type. Upper panels: Northern blots after probing with labeled dappled. Lower panels: control for loading of RNA in each lane by reprobing the blots with labeled $r p 49$. a Adult total RNA from Oregon R (lane 1), dpld ${ }^{M L B} / d p l d^{E J L}$ transheterozygotes (lane 2), and $d p l d^{M L B}$ homozygotes (lane 3). b Embryonic total RNA from Oregon R (lane 1) and $d p l d^{01857}$ homozygotes (lane 2); embryonic poly-A RNA from Oregon R (lane 4), and $d p l d d^{E J L}$ homozygotes (lane 5). Lane 3 contains no RNA. Embryos from the homozygous lethal strains $d p l d^{E J L}$ and $d p l d^{01857}$ were sorted via a Balancer chromosome expressing GFP ( $C y O, K r$-GFP), in order to select for the homozygous mutant embryos. Quantitation after correcting for loading using rp49 shows reduced levels of dappled RNA in all mutants. Compared with wild type, approximate levels are (a) $65 \%$ in lane 2 and $80 \%$ in lane 3; (b) $40 \%$ in lane 2 and $10 \%$ in lane 5 
Based on sequence homology from our alignments and the Flybase Genome Annotation, Drosophila gene CG2140/dappled encodes a microsomal cytochrome b5 (Fig. 1; http://flybase.org/reports/FBgn0033189.html). Genome annotation has identified CG2140 as the only cytochrome b5 in Drosophila. One related Drosophila sequence has been identified, cytochrome b5-related (Cyt-b5-r; http:// flybase.org/reports/FBgn0000406.html). Cyt-b5-r has 436 residues (there are only 134 for Dappled and 135 for human cytochrome b5), and only 30 of the Cyt-b5-r residues are identical to the Dappled sequence. In a comparison of the dappled encoded amino acid sequence with a selection of other cytochrome b5 proteins from mammals and insects, Dappled has $45 \%$ identity with human cytochrome b5 and $76 \%$ identity with housefly cytochrome b5 (Fig. 3). Overall, the selected mammals and insects have $32 \%$ identity. Cytochrome b5 is a highly conserved, ubiquitous family of small hemoproteins that participate in electron transfer in multiple biochemical reactions. These reactions are involved in many biological processes, such

Rattus_norvegicus
Mus_musculus
Sus_scrofa
Homo_sapiens
Drosophila_dappled
Musca_domestica
Anopheles_gambiae
Prim.cons.

Rattus_norvegicus Mus_musculus

Sus_scrofa

Homo_sapiens

Drosophila_dappled

Musca_domestica

Anophēles_gambiae

Prim.cons.

Rattus norvegicus Mus_musculus

Sus scrofa

Homo_sapiens

Drosophila dappled

Musca_domestica

Anophēles_gambiae

Prim.cons.

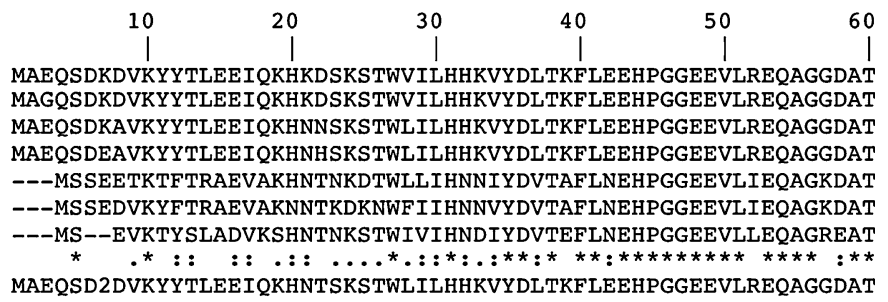

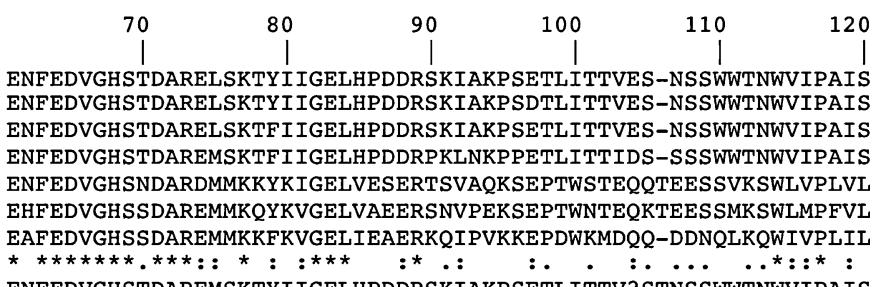
ENFEDVGHSTDAREMSKTYIIGELHPDDRSKIAKPSETLITTV2STNSSWWTNWVIPAIS

130
ALVVALMYRLYMAED--
ALAVALMYRLYMAED--
ALVVSLMYHFYTSEN--
AVAVALMYRLYMAED--
CLVATLFYKFFFGGAKQ
GLVATLIYKFFFGTKSQ
GLLATILYRFYFTQ---
$:$ : : : : : :
ALVV2LMYRFY2AED2Q

Fig. 3 Cytochrome b5 sequences. The sequences of Dappled and selected other cytochrome b5 proteins from insects and mammals are compared using Clustal W for alignment and consensus generation (Prim. Cons.). See Wang et al. (2007) for an X-ray crystal structure comparison of the heme binding domain, residues 6-95. Note the high sequence identity within this region, which is $82 \%$ for residues $40-73$. Sequence accession numbers are Rattus norvegicus GenBank AAB67610.1; Mus musculus Swiss-Prot P56395.2; Sus scrofa Swiss-Prot P00172.3, Homo sapiens GenBank AAA35729.1; Drosophila dappled GenBank HQ864711; Musca domestica GenBank AAA56985.1; Anopheles gambiae GenBank AAO24766.1 
as conversion of methemoglobin, sterol metabolism, fatty acid desaturation, and cytochrome P450-catalyzed reactions (Schenkman and Jansson 2003).

Hemocyte Misregulation and Melanotic Nodule Formation in dappled Mutants

The $d p l d^{M L B}$ insertion has made analysis possible, as in addition to producing consistent melanotic tumor formation, it is the only viable allele. Maintenance of $d p l d^{M L B}$ homozygotes as a stock, however, was not feasible because of effects on fertility. Homozygous $d p l d^{M L B}$ or transheterozygous $d p l d^{M L B} / d p l d^{E J L}$ females and males produced few progeny, even when mated with wild-type flies $(<5$ progeny/ dappled parent), and mutant females laid few eggs ( $\sim 50 /$ female). It is unlikely that this fertility problem is due simply to physical obstruction in the abdomen, because flies with less obtrusive tumors (i.e., small tumors near the thorax) also show low fertility. To avoid the effects of accumulation of second site mutations by maintaining the $d p l d^{M L B}$ chromosome over a balancer, all experiments were done on progeny from crosses that produced transheterozygotes or hemizygotes.

The development of the Gal4-UAS system (Brand and Perrimon 1993) to include drivers for the hematopoietic system makes it more feasible to be able to investigate the blood cell phenotypes of mutant genes, for example, Kimbrell et al. (2002) and Zettervall et al. (2004). The hemese-Gal4 construct drives expression in blood cells of the plasmatocyte and lamellocyte classes (Zettervall et al. 2004). Strains with hemese-Gal4 UAS-GFP thus have the blood cells marked with GFP, enabling hemocyte counting and phenotype analysis in various genetic strains (Zettervall et al. 2004). We used hemese-Gal4 UAS-GFP to investigate the blood cells of dappled melanotic tumor mutants. In melanotic tumors of $d p l d^{E J L} / d p l d^{M L B}$; hemeseGal4 UAS-GFP larvae, all of the cells in the masses were found to fluoresce strongly for GFP (Fig. 4). Cell counts of circulating plasmatocytes and lamellocytes showed large numbers of circulating lamellocytes (Fig. 4). Lamellocytes are not found in normal, healthy larvae, whereas in melanotic nodule mutants lamellocytes are abundant (Crozatier and Meister 2007). The dappled melanotic tumors are thus specifically designated as melanotic nodules, which consist of hemocytes, as opposed to melanizations, which are non-blood cell tissues that have melanized (Minakhina and Steward 2006b).

Mutant dappled larvae were categorized based on the relative size of the melanotic nodules. Larvae with small versus large melanotic nodules showed different numbers of circulating plasmatocytes and lamellocytes (Fig. 4). Notably, lamellocytes are more prevalent in small tumors, whereas plasmatocytes are more abundant in large nodules. One possible interpretation of the higher number of lamellocytes in smaller nodules is that these nodules are still actively forming, and lamellocytes are still being recruited to the nodules. During normal larval development, the number of plasmatocytes typically increases two- to threefold from the early wandering (early) to the late wandering (late) stage of the third instar (Crozatier and Meister 2007; Zettervall et al. 2004). Plasmatocyte representation from early to late stage wandering was different for small and large tumors. Larvae with large tumors showed an increase from early to late stage, whereas larvae with 

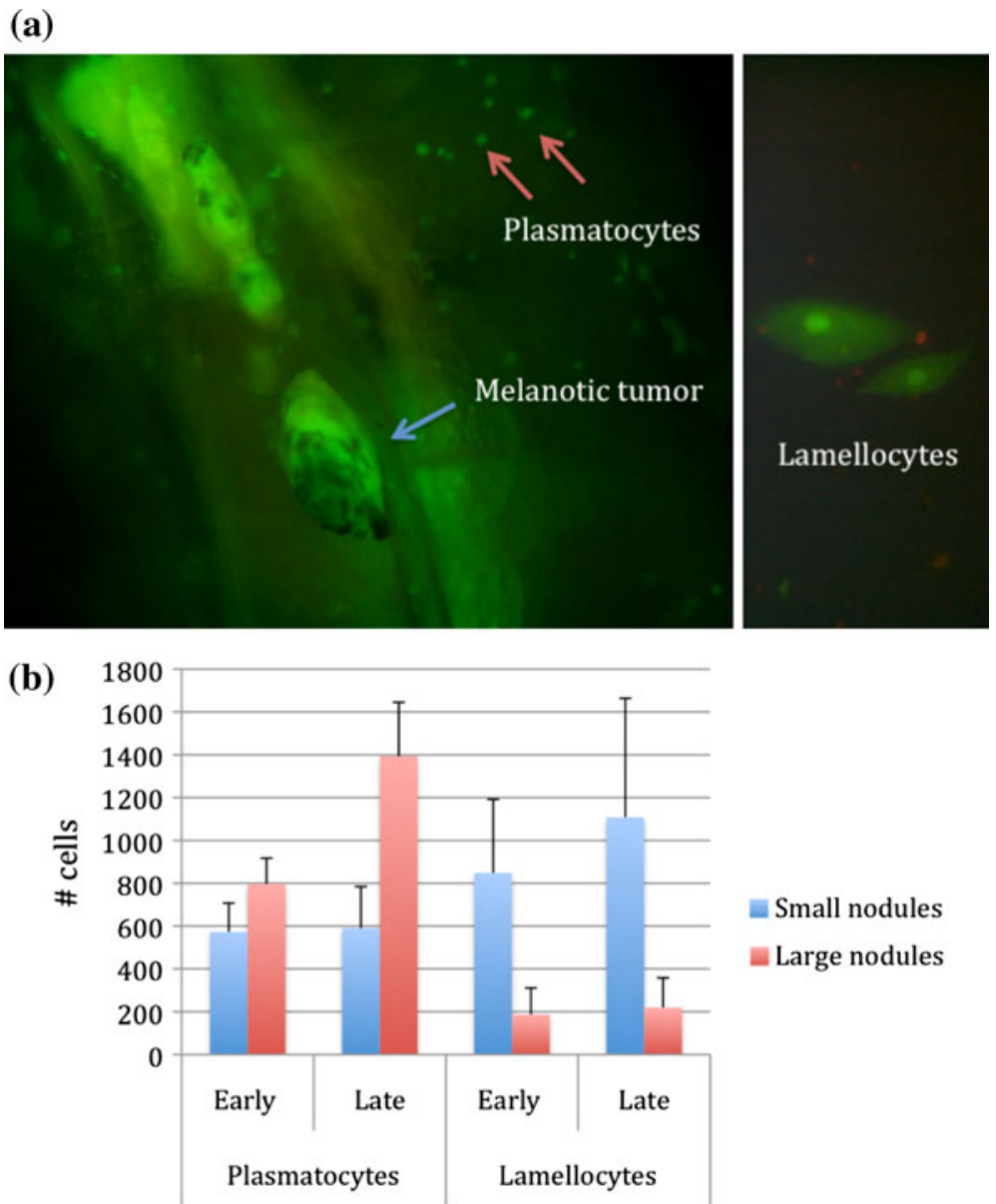

Fig. 4 Hemocytes of dappled viable mutants. a Hemocytes visualized by GFP expression show (left) melanotic tumors/nodules in a third-instar larva as clumps of hemocytes that are partially melanized, along with circulating plasmatocytes, and (right), at higher magnification, lamellocytes. Lamellocytes are approximately four times larger than plasmatocytes. GFP expression is driven by the hemese-Gal4 driver. b Cell counts of circulating hemocytes in early and late wandering third-instar larvae show high levels of lamellocytes and characterize the dynamic process of melanotic tumor formation by comparing mutants with small (left bars) versus large (right bars) melanotic nodules. Error bars show 0.95 confidence limits. $N \geq 15$ for each category. (Color figure online)

small tumors did not (Fig. 4), and this may also reflect the dynamic nature of dappled melanotic nodule formation.

Heterozygosity of dappled does not produce tumors, but it does have a dominant effect on blood cell number. The dappled heterozygotes (Bal/dpld) have a significantly higher number of plasmatocytes than wild-type larvae $(+/+)$. This difference was confirmed by counting hemocytes of dappled heterozygotes $(\mathrm{dpld} /+)$ and their control siblings (Bal/+) (Fig. 5). Further, counts of siblings showed that the increase in plasmatocytes is present both early and late in the third instar. The 

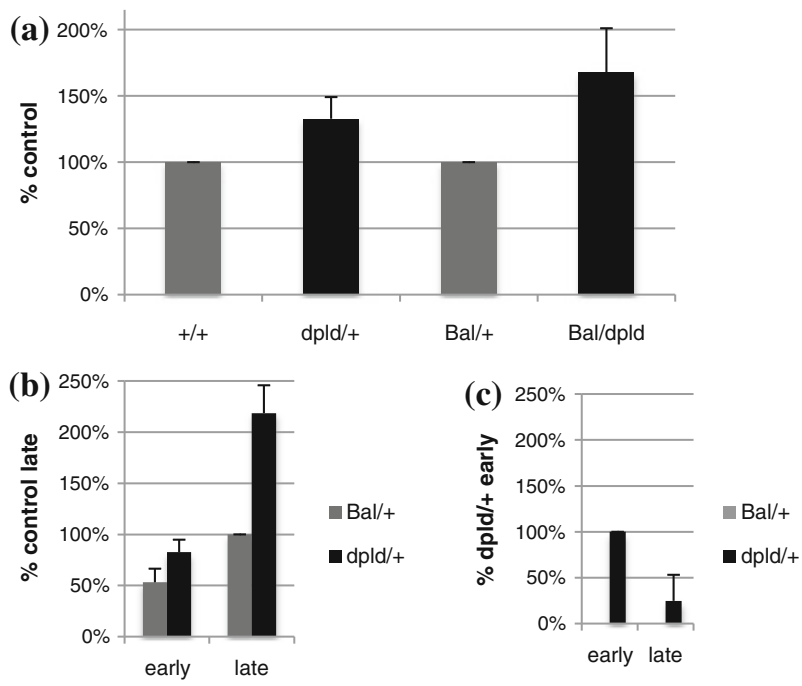

Fig. 5 Plasmatocytes and lamellocytes are increased in dappled heterozygotes. a Cell counts of plasmatocytes during late third instar of dappled heterozygotes, over a Balancer (Bal) or wild-type $(+)$ chromosome, show significantly more plasmatocytes than wild-type $(+/+)$ and Balancer $(\mathrm{Bal} /+)$ controls. Counts were normalized across three experiments to the percentage of controls. The average for control $+/+$ was 5,697 cells/larva and $\mathrm{Bal} /+$ was 4,935 cells/larva. $P<0.01$ for dpld/+ versus $\mathrm{Bal} /+$, and $P<0.05$ for Bal/dpld versus $+/+$. b Cell counts of plasmatocytes of dappled heterozygotes and controls that are siblings show that the increase in plasmatocytes has already occurred by early wandering third instar. Additionally, dappled heterozygotes show a normal increase, which is approximately two- to threefold, in plasmatocyte number from early to late stage. Counts were normalized across three experiments to the percentage of control Bal/+ late, which averaged 4,125 cells/larva. $P<0.01$ for both early and late of dpld/+ versus Bal/+. c Cell counts of the larvae (b) show that lamellocytes are also increased. Control Bal/+ larvae had no lamellocytes in any sample. $P<0.05$ for both early and late dpld/ + versus $\mathrm{Bal} /+$. The average number of cells in early dappled heterozygotes was 5 , with high variability and no significant difference from late dappled heterozygotes $(P>0.05) . N \geq 15$ per genotype for each experiment

relative numbers of lamellocytes in dappled heterozygotes is less clear, as the average was very low, many larvae had no lamellocytes, and the number did not increase from early to late stage (Fig. 5). Thus, although the lamellocyte data are statistically significant $(P<0.05)$, dappled heterozygosity at most yields a very weak induction of lamellocyte differentiation.

\section{Rescue of Hemocyte Phenotype but Not Lethality by Wild-Type dappled in Blood Cells}

We next asked if the dappled phenotype could be rescued by expressing wild-type dappled only in blood cells. We constructed a plasmid containing UAS-dpld, which was used to make transgenic strains with $U A S$-dpld in different chromosomal locations. The hemese-Gal4 was then used to drive UAS-dpld, and the rates of melanotic nodule formation were compared in dappled mutants and controls. In addition to hemocytes, hemese-Gal4 drives strong expression in the salivary glands, 
Table 1 Melanotic tumor phenotype is rescued by dappled expression in hemocytes

\begin{tabular}{|c|c|c|c|c|c|}
\hline \multirow[t]{2}{*}{ Cell type } & \multirow[t]{2}{*}{ Gal4; UAS } & \multicolumn{3}{|c|}{ Percentage $(n)$ of adults ${ }^{\mathrm{a}}$ with melanotic nodules } & \multirow[t]{2}{*}{ Total $N$} \\
\hline & & $\begin{array}{l}\text { Negative } \\
\text { control }^{\mathrm{b}}\end{array}$ & $\begin{array}{l}\text { Positive } \\
\text { control }^{\mathrm{c}}\end{array}$ & Rescue test ${ }^{\mathrm{d}}$ & \\
\hline \multirow[t]{3}{*}{ Blood cells } & hemese-Gal4; UAS-dpld line 1 & $0 \%(352)$ & $87.5 \%(32)$ & $5 \%(121)$ & 505 \\
\hline & $\begin{array}{l}\text { hemese-Gal4; UAS-dpld } \\
\text { line } 3 \mathrm{a}\end{array}$ & $0 \%(526)$ & $94.1 \%(43)$ & $7.5 \%(105)$ & 674 \\
\hline & $\begin{array}{l}\text { hemese-Gal4; UAS-dpld } \\
\text { line } 9 \mathrm{~b}\end{array}$ & $0 \%(353)$ & $93 \%(17)$ & $5.7 \%(106)$ & 476 \\
\hline Control & sgs3-Gal4; UAS-dpld & $0 \%(514)$ & $89.3 \%(75)$ & $81.6 \%(103)$ & 692 \\
\hline \multicolumn{6}{|c|}{$\begin{array}{l}\text { a Adults are progeny from crosses of } w ; C y O / d p l d M L B ; \text { Gal4 females with } w / Y ; C y O / d p l d E J L ; T M 3, S b \\
\text { or } U A S \text { - } d p l d / U A S \text { - } d p l d \text { males }\end{array}$} \\
\hline \multicolumn{6}{|c|}{ b All genotypes with $C y O / d p l d M L B$ or $E J L$. Two thirds of the progeny are expected to be in this category } \\
\hline \multicolumn{6}{|c|}{${ }^{\mathrm{c}} d p l d$ transheterozygotes with Gal4 or $U A S$} \\
\hline \multicolumn{6}{|c|}{${ }^{\mathrm{d}} d p l d$ transheterozygotes with both Gal4 and $U A S$} \\
\hline \multicolumn{6}{|c|}{${ }^{\mathrm{e}}$ Results with all three $U A S$-dpld lines } \\
\hline
\end{tabular}

so a control salivary gland driver, sgs3-Gal4, was also used to drive UAS-dpld. To test for rescue of lethality by ubiquitous expression, the $d a$-Gal4 driver (Pili-Floury et al. 2004) was used.

The dappled mutants with hemese-Gal4, but not sgs3-Gal4, driving UAS-dpld are rescued from the melanotic phenotype. Three different UAS-dpld insertions were tested with hemese-Gal4, and all showed similar rates of rescue (Table 1). Dissections were performed to determine if blood cell nodules might be forming but not melanizing. The dissections confirmed that blood cell nodule formation without accompanying melanization did not occur.

The dominant effect of heterozygous dappled on plasmatocyte number is also rescued by adding dappled expression to blood cells. Hemocyte counts of dappled heterozygotes with hemese-Gal4 driving UAS-dpld had, on average, reduced numbers of hemocytes, compared with control dappled heterozygotes (Fig. 6). The number of lamellocytes in heterozygotes was also reduced on average, but with the small number and variability in lamellocytes, was not significantly different (Fig. 6). These results do not necessarily rule out other tissues as important for the dappled mutant blood cell phenotypes, but rather emphasize that the level of dappled expression in the blood cells regulates number and differentiation.

Expression of wild-type dappled in blood cells did not rescue the mutant lethality. In contrast, ubiquitous expression of wild-type dappled completely rescued the mutant lethality, producing normal adults with no tumors (Table 2). These results are consistent with the aberrant tissue morphology of lethal dappled (Rodriguez et al. 1996) and establish the importance of dappled transcript levels for normal function in other tissues. 

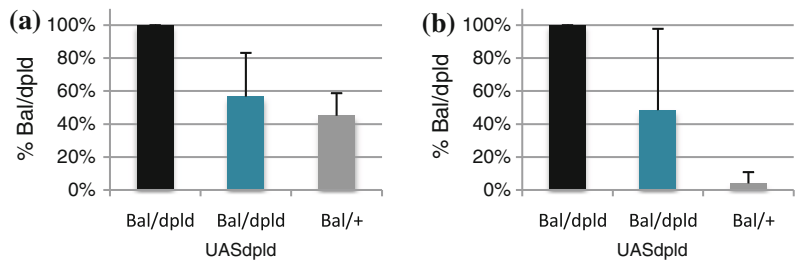

Fig. 6 Rescue of dappled heterozygote phenotype by expression of wild-type dappled in hemocytes. The dappled heterozygotes with UAS-dpld driven by hemese-Gal4 (Bal/dpld UAS-dpld) were compared with controls without UAS and Gal4 (Bal/dpld and $\mathrm{Bal} /+$ ). Counts were normalized across three experiments to the percentage of Bal/dpld. Plasmatocyte counts (a) are significantly reduced in rescued larvae compared with Bal/dpld $(P<0.05)$, but not compared with Bal/ $+(P>0.05)$. Lamellocyte counts $(\mathbf{b})$ are reduced on average in rescued larvae, but not to a significant extent $(P>0.05$, compared with both Bal/ dpld and $\mathrm{Bal} /+$ ). $N \geq 15$ per genotype for each experiment

Table 2 Hemocyte expression of dappled is insufficient for rescue of lethality

\begin{tabular}{lllll}
\hline Expression type & Gal4; UAS & \multicolumn{2}{l}{ Percentage $(n)$ survival of adults $^{\mathrm{a}}$} & \multirow{2}{*}{ Total N } \\
\cline { 3 - 4 } & & Control $^{\mathrm{b}}$ & Rescue test $^{\mathrm{c}}$ & \\
\hline Ubiquitous & daughterless-Gal4; UAS-dpld & $84.6 \%(1503)$ & $15.4 \%(273)$ & 1776 \\
Blood cells & hemese-Gal4; UAS-dpld & $100 \%(1086)$ & $0 \%(0)$ & 1086 \\
\hline
\end{tabular}

\footnotetext{
${ }^{a}$ Adults are progeny from crosses of $w$; CyO/dpldEJL;TM3,Sb or UAS-dpld/UAS-dpld females with $w / Y$; CyO/Df1888;TM3,Sb/Gal4 males

b $C y O / d p l d E J L$ or $C y O / D f(2 R) S T 1$ with Gal4 or $U A S .85 \%$ of the total progeny are expected to be in this category

${ }^{c}$ dpldEJL/Df(2R)ST1 with both Gal4 and UAS. $15 \%$ of the total progeny are expected to be in this category
}

\section{Effect of dappled on Hematopoietic Waves During Development}

The dappled nodules form early in larval development, which is more consistent with an increase of plasmatocytes from the first hematopoietic wave (embryonic origin) rather than the second hematopoietic wave (lymph gland origin at the end of the third instar). As noted above, plasmatocytes in dappled tumors express the hemese-Gal4 driver, which does not express in the lymph glands, further indicating an early developmental origin for these cells. Additionally, the lymph glands appear normal, at least at a gross level, and only one dappled enhancer trap expresses in the lymph glands (Rodriguez et al. 1996). We analyzed $1 \mathrm{~kb}$ of the dappled upstream region for potential binding by blood cell, lymph gland, and other transcription factors using the Transfac database (Wingender 2008) provided by Biobase Biological Databases (http://www.biobase-international.com/index.php?id=home). Among the transcription factor binding sites are many that are predicted to be $\mathrm{T}$ cell factor/pan binding sites (Brunner et al. 1997), consistent with blood cell expression, and tinman/Nkx2.5 binding sites (Kimbrell et al. 2002), suggesting lymph gland expression (Table 3). 
We tested for dappled effects in both hematopoietic waves by counting plasmatocytes at a time in development when cells of both hematopoietic waves are present. Cells of the two waves were differentiated by (1) using hemese-Gal4 to drive $U A S-G F P$ in cells from the first wave of development, so that first-wave cells fluoresced green, and (2) staining all hemocytes with monochlorobimane dye, so that the second-wave hemocytes fluoresced blue but not green. The developmental stage selected to have both sets of hemocytes was white prepupae, age $0-3 \mathrm{~h}$ postformation as white prepupae. These results show that heterozygosity of dappled increases the number of plasmatocytes from both hematopoietic waves (Table 4).

Mutation of dappled affects both sexes similarly, and our results show both sexes together (Figs. 4, 5, 6). Similar numbers of the sexes are needed per sample,

Table 3 Predicted T cell factor/ pangolin and Nkx 2.5/tinman sites

Predicted by Transfac

Professional Match 2009.3, provided by Biobase Biological Databases

\begin{tabular}{|c|c|}
\hline \multicolumn{2}{|c|}{$\mathrm{T}$ cell factor/pangolin } \\
\hline-730 & (+) 1.0000 .994 gcgtaTCAAAagcaca TCF \\
\hline-687 & (-) 0.9640 .946 gettagTTTGTtgtta TCF \\
\hline-611 & (-) 0.9280 .919 gactgtTTTTAttgaa TCF \\
\hline-440 & (-) 0.9280 .929 atacatTTTTAttaat TCF \\
\hline-430 & (+) 0.9280 .922 attaaTGAAAtggtct TCF \\
\hline-408 & (-) 0.9570 .938 aataaaTTTGCttacg TCF \\
\hline-395 & (-) 0.9640 .948 acgaatTTTGTttgta TCF \\
\hline-383 & (-) 0.9280 .922 tgtacaTTTCAtttga TCF \\
\hline-170 & (+) 0.9640 .944 tgtaaACAAAttataa TCF \\
\hline \multicolumn{2}{|c|}{ Nkx 2.5/tinman } \\
\hline-939 & (-) 0.8010 .786 tgattgAAGTTggcaa NKX2.5 \\
\hline-935 & (+) $0.8690 .828 \operatorname{tg}$ AAGTT NKX2.5 \\
\hline-923 & (-) 0.8690 .870 ААCTTaа NKX2.5 \\
\hline-896 & (+) 1.0000 .859 cccgtCACTTgaatta NKX2.5 \\
\hline-894 & (+) 1.0000 .971 cgtCACTTga NKX2.5 \\
\hline-891 & (-) 1.0001 .000 CACTTga NKX2.5 \\
\hline-795 & (+) 0.8440 .805 taggcCACTCcaggat NKX2.5 \\
\hline-780 & (+) $0.8000 .819 \operatorname{tgcCAATTgc~NKX2.5}$ \\
\hline-761 & (-) 0.8690 .823 AACTTac NKX2.5 \\
\hline-711 & (+) 1.0000 .741 gccgtCACTTatcaat NKX2.5 \\
\hline-709 & (+) 1.0000 .906 cgtCACTTat NKX2.5 \\
\hline-706 & (-) 1.0000 .939 CACTTat NKX2.5 \\
\hline-677 & (+) 0.8010 .732 ttgttAACTTgaattg NKX2.5 \\
\hline-672 & (-) 0.8690 .884 AACTTga NKX2.5 \\
\hline-551 & (+) $0.8690 .828 \operatorname{tg}$ AAGTC NKX2.5 \\
\hline-429 & (-) 0.7880 .751 ttaatgAAATGgtctg NKX2.5 \\
\hline-377 & (+) 0.8000 .815 tttCATTTga NKX2.5 \\
\hline-374 & (-) 0.7830 .808 CATTTga NKX2.5 \\
\hline-235 & (+) 0.8330 .818 tgatcCACTAtacaat NKX2.5 \\
\hline-145 & (+) 0.8690 .823 atAAGTT NKX2.5 \\
\hline
\end{tabular}


Table 4 Hemocyte number differs by sex during development in wild type and dappled

\begin{tabular}{|c|c|c|c|c|}
\hline \multirow[t]{2}{*}{ Genotype } & \multirow[t]{2}{*}{ Sex } & \multicolumn{3}{|c|}{ Number of hemocytes $( \pm 95 \%$ confidence interval) } \\
\hline & & $\begin{array}{l}\text { Average for } \\
\text { embryonic origin }\end{array}$ & $\begin{array}{l}\text { Average for lymph } \\
\text { gland origin }\end{array}$ & Total cells \\
\hline \multirow[t]{3}{*}{ Wild type } & Male & $3380( \pm 753)$ & $4610( \pm 942)$ & $7990( \pm 1237)$ \\
\hline & Female & $5413( \pm 1492)$ & $6956( \pm 1505)$ & $12369( \pm 2109)$ \\
\hline & $P$, male vs. female & $<0.01$ & $<0.001$ & $<0.01$ \\
\hline \multirow[t]{3}{*}{$d p l d^{\mathrm{a}}$} & Male & $6455( \pm 1128)$ & $6675( \pm 1268)$ & $13130( \pm 1716)$ \\
\hline & Female & $8628( \pm 1879)$ & $8987( \pm 1374)$ & $17615( \pm 3013)$ \\
\hline & $P$, male vs. female & $<0.05$ & $<0.01$ & $<0.01$ \\
\hline \multirow[t]{2}{*}{ Wild type vs. $d p l d^{\mathrm{a}}$} & $P$, male vs. male & $<0.001$ & $<0.01$ & $<0.001$ \\
\hline & $P$, female vs. female & $<0.01$ & $<0.05$ & $<0.01$ \\
\hline
\end{tabular}

${ }^{\text {a }}$ dpld heterozygotes, dpldEJL/+

however, as the sexes differ in the number of hemocytes. For the experiment depicted in Table 4, we separated the sexes in order to demonstrate this finding and test for a sex difference in the second hematopoietic wave as well. The results are that in both wild type and dappled there is a significant difference between males and females in the number of hemocytes for both hematopoietic waves.

Curcumin Enhancement of the dappled Melanotic Phenotype

Cytochrome b5 is widely studied for its effects on drug metabolism, and conversely, various drugs are investigated for alteration of cytochrome b5 levels (El-Lakkany et al. 2004; Juvonen et al. 1992; Portoles et al. 1989; Schenkman and Jansson 1999; Sheweita 2004; Yamaori et al. 2003; Yamazaki et al. 2002). We hypothesized that modulation of the dappled phenotype could be used as an in vivo assay for cytochrome b5 related effects of endogenous and xenobiotic compounds. We are therefore screening compounds with a known connection to cytochrome P450s and/ or anti-inflammatory and anticarcinogenic properties for enhancement or suppression of the dappled melanotic phenotype. Initial studies with aspirin and the corticosteroid dexamethasone are negative (data not shown), whereas curcumin, the active ingredient in the spice turmeric, has consistently shown an enhancement of the melanotic phenotype (Table 5; Fig. 7). Adult dappled mutants that have been raised (egg to adult) on curcumin-supplemented food have an enhanced melanotic phenotype, showing more and/or larger melanotic tumors (Table 5). The enhanced phenotype of the dappled mutants is already evident in the larval stages (Fig. 7). Adult dappled heterozygotes, however, do not develop a melanotic phenotype when raised on curcumin (Table 5). Since both the heterozygotes and heteroallelic mutants have some reduction in dappled transcript levels, the effects of curcumin may depend on the exact amount of cytochrome b5 protein that is produced. 
Table 5 Curcumin enhances the melanotic tumor phenotype of dappled

\begin{tabular}{|c|c|c|c|c|c|c|c|c|}
\hline \multirow[t]{3}{*}{ Diet } & \multicolumn{8}{|c|}{ Percentage of adults ${ }^{\mathrm{a}}$ with melanotic tumors } \\
\hline & \multicolumn{2}{|c|}{ Genotype $^{\mathrm{b}}(N)$} & \multicolumn{6}{|c|}{ Melanotic nodule severity $(\%)^{\mathrm{c}}$} \\
\hline & Balancer & dpld & 1 & 2 & 3 & 4 & 5 & 6 \\
\hline Normal & $0 \%(373)$ & $90.3 \%(154)$ & 0.6 & 3.9 & 59.1 & 25.3 & 1.3 & 9.7 \\
\hline Curcumin supplement & $0 \%(762)$ & $87.1 \%(310)$ & 1.6 & 26.8 & 56.1 & 1.3 & 1.3 & 12.9 \\
\hline
\end{tabular}

a Adults are progeny from crosses between $w ; C y O / d p l d M L B$ and $w ; C y O / d p l d E J L$

b "Balancer" indicates heterozygous CyO/dpldMLB or EJL. "dpld" indicates transheterozygous dpldMLB/dpldEJL

c Severity decreases left to right. Category 1 is defined as multiple small nodules; 2 , single large or $>1$ typical nodule; 3 , typical nodule; 4 , small nodule; 5 , very small nodule; 6 , no nodule

\section{Discussion}

We have identified dappled as a member of the conserved, ubiquitous cytochrome b5 family. The complexities of cytochrome b5 function, through its participation in electron transfer in multiple biochemical reactions, are reflected in the difficulties and limited study of its genetics in higher organisms. Characterizations in humans have shown that cytochrome b5 mutation causes methemoglobinemia, which interferes with the balance between hemoglobin and methemoglobin, thus affecting oxygenation and inflammation (Hegesh et al. 1986; Umbreit 2007). Mutation of cytochrome b5 reductase also causes methemoglobinemia and is more common (Davis et al. 2004). In addition, one case of cytochrome b5 methemoglobinemia may also have been associated with hormonal defects (Giordano et al. 1994). With the exception of a porcine mutation associated with an altered androstenone level (Lin et al. 2005), cytochrome b5 mutations in other organisms have not been identified. In mice, cytochrome b5 deletion is also lethal, but experiments with a tissue-specific knockout have allowed liver function to be addressed (Finn et al. 2008). With our studies of dappled, Drosophila now provides a tractable genetic model for the study of cytochrome b5 in blood cells and other tissues.

Hemocytes are particularly sensitive to mutation of dappled and show that cytochrome b5 functions to produce normal differentiation and numbers of hemocytes. Mutation of one copy of dappled results in a significant increase in the number of circulating hemocytes, and mutation of both copies results in the formation of melanotic nodules. The formation of dappled melanotic nodules is a Class 2 reaction in the classification of Watson et al. (1991), in which Class 2 represents alteration of immune cells and Class 1 represents normal immune cells responding to other cell types that are aberrant. The dominant effect on blood cell number emphasizes that wild-type levels of cytochrome b5 are needed for full normal functions. In humans, levels of cytochrome b5 have been implicated as important with respect to xenobiotic metabolism and health. Kurian et al. (2007) hypothesized that functionally significant genetic variability in cytochrome b5 occurs in individuals without clinical symptoms. They investigated processing of hydroxylamines, which are oxidative products of arylamine drugs and 

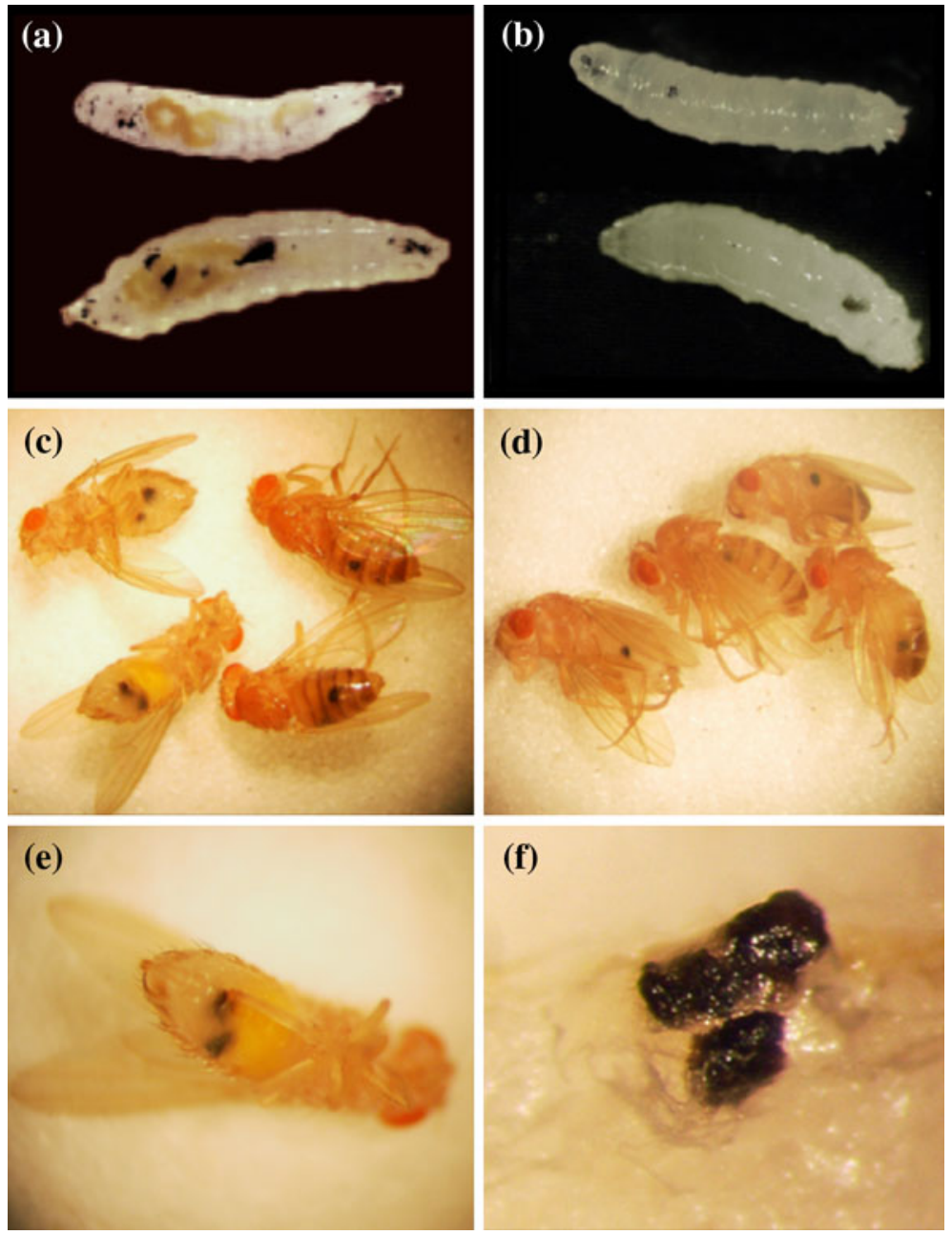

Fig. 7 Curcumin enhances the dappled melanotic phenotype. The dappled mutants on the left (a, c, and e) were raised on curcumin-supplemented food. Those on the right (b and $\mathbf{d}$ ) were raised on normal food. f Free-floating melanotic tumors from a curcumin-fed adult. (Color figure online)

environmental carcinogens, by the mutant cytochrome b5 of one heterozygous individual and found, in vitro, altered affinity for hydroxylamine substrates and dramatically reduced cytochrome b5 expression (Kurian et al. 2007).

Expression of wild-type dappled in hemocytes rescued both the increase in blood cell number and the formation of melanotic nodules. The driver used was hemeseGal4, which is active in circulating plasmatocytes and sessile hemocytes, but not the lymph glands (Markus et al. 2009; Zettervall et al. 2004). Interestingly, the sessile hemocytes are a newly discovered hematopoietic site and appear to be the main developmental origin of lamellocytes (Markus et al. 2009). This site of expression may be the key factor in rescuing the dappled melanotic nodule phenotype. Rescue 
of the plasmatocyte increase in dappled heterozygotes is expected to result from the hemese-Gal4 expression in circulating blood cells of the first hematopoietic wave, both because of the age of the larvae (prerelease of lymph gland cells) and because hemese-Gal4 does not express in lymph glands. However, dappled also increased the number of hemocytes from the second hematopoietic wave. Hemocytes from both developmental origins, embryonic and lymph gland, require dappled in order to be produced in normal amounts. It is an interesting question as to whether the plasmatocyte increase in dappled mutants can be independently controlled in the first and second hematopoietic waves.

Does the increase in plasmatocytes indicate overproliferation or less apoptosis? Since dappled encodes a cytochrome b5, the obvious choice would be apoptosis rather than overproliferation. There are two problems with this choice: it is mitochondrial cytochrome b5 that is established in the canonical cytochrome $\mathrm{C}$ signaling apoptotic pathway, and the use of that pathway by Drosophila is controversial (Kornbluth and White 2005; Means and Hays 2007; Oberst et al. 2008; Ow et al. 2008). Microsomal cytochrome b5, however, has also been suggested to be involved in cytochrome C mediated apoptosis (Davydov 2001), and Drosophila may have its own version of apoptotic signaling that uses the dappled cytochrome b5. Further, blood cells are well described for their role in response to apoptotic cells, but the role of their own apoptosis in development and infection is not clear (Bruckner et al. 2004; Crozatier and Meister 2007; Lemaitre and Hoffmann 2007).

The sensitivity of hemocytes (compared with other cell types) to dappled mutation may result from blood cell mitotic cell division continuing during development (Crozatier and Meister 2007). There are many potential mechanisms for dappled to affect overproliferation, especially given the increasingly complex functions of plasmatocytes (Charroux and Royet 2009). One option consistent with cytochrome b5 function, and potentially the dappled effects on fertility, is that hormonal signaling of hematopoiesis is affected. In that case, however, rescue of the dappled blood cell phenotype would be predicted by adding back wild-type dappled expression in the steroid-producing ring gland (Gilbert 2004) rather than hemocytes. Alternatively, dappled expression could rescue a steroid-processing step in the hemocytes.

An intriguing option is that cytochrome b5 has a new, previously unidentified role in blood cell signaling pathways, for example, through JAK/Stat or JNK. More specifically, a candidate to mediate the effects of dappled is TNF-receptor associated factor 6 (Traf6), which is predicted from protein studies to interact with cytochrome b5 (Drosophila Interactions Database, http://www.droidb.org). Among its activities, Traf6 is an upstream activator of nuclear factor-kappaB, and mutants are immune response defective (Cha et al. 2003; Wu and Arron 2003). Cytochrome b5 involvement with Traf6 is a testable hypothesis through analysis of genetic interaction between dappled and Traf6 mutants.

Our results with curcumin demonstrate that screening of endogenous and xenobiotic compounds for effects on the dappled phenotypes can be done as an in vivo approach for analysis of cytochrome b5. Curcumin is widely studied for its potential as a nutritional pharmaceutical (Duvoix et al. 2005; Jagetia and Aggarwal 2007). Among its many activities, which are mainly anti-inflammatory and 
anticancer, curcumin inhibits cytochrome P450s, down-regulates NFkB, and affects apoptosis and the cell cycle (Alpers 2008; Appiah-Opong et al. 2007; Kwon et al. 2007; Sa and Das 2008). Curcumin enhanced the melanotic phenotype of dappled mutants. This effect of curcumin is consistent with NFkB involvement, but there are other alternatives, in particular, cytochrome $\mathrm{P} 450$ processing in the fat body.

Mutation of dappled showed similar effects in females and males, but an unexpected consequence of our studies is the finding that females and males differ significantly in the number of circulating blood cells. In humans, a difference between the sexes in the numbers of blood cell types is well established, but the effects are unclear (Blum and Pabst 2007; Huppert et al. 1998; Reichert et al. 1991). In both humans and Drosophila, there are differences in the immune responses between the sexes that affect health (Dale et al. 2006; Marriott and Huet-Hudson 2006; Shames 2002; Taylor and Kimbrell 2007; Ye et al. 2009; Zandman-Goddard et al. 2007), and the blood cell number differential may be part of mediating the immune response differences. Recently, Drosophila hemocytes have been shown to directly signal immune induction of the fat body, and a reduced number of hemocytes resulted in less immune induction (Shia et al. 2009). In Drosophila, the increase in hemocytes of dappled and females may result in higher immune induction, which may or may not provide a healthier condition. For example, human females typically have a more robust immune response but also a higher incidence of autoimmune disorders (Dale et al. 2006; Zandman-Goddard et al. 2007).

Cytochrome b5 expression is critical for survival, and its importance to hemocytes and additional tissues is emphasized by the finding that ubiquitous, but not hemocyte, expression of wild-type dappled rescued lethality. The functions of cytochrome b5 are open for testing in a wide range of tissue types using dappled. In all respects, genetic interaction and drug screens for suppressors and enhancers of dappled will be useful ways to discern the many roles of cytochrome b5 function.

Acknowledgments We thank the Bloomington Stock Center for fly strains and Dan Hultmark for the hemese-Gal4 stock. We are grateful to Kathleen Beckingham, Jeanette Natzle, Ylva Engstrom, and John Kiger for discussions and comments on the manuscript. We are also grateful to Chyong-Yi Wu and Edward Yonter for early work on plasmid rescue and fertility, Katherine Taylor for assistance with tumor rescue tests, and Genetic Services, Inc., for transgenic strains. This work was supported by grants NIH R01GM61458, NASA NNA04CC76A, and American Cancer Society IM697B/9302006CIM to DAK.

Open Access This article is distributed under the terms of the Creative Commons Attribution Noncommercial License which permits any noncommercial use, distribution, and reproduction in any medium, provided the original author(s) and source are credited.

\section{References}

Aggrawal K, Silverman N (2007) Peptidoglycan recognition in Drosophila. Biochem Soc Trans 35:1496-1500

Alpers DH (2008) The potential use of curcumin in management of chronic disease: too good to be true? Curr Opin Gastroenterol 24:173-175

Appiah-Opong R, Commandeur JN, van Vugt-Lussenburg B, Vermeulen NP (2007) Inhibition of human recombinant cytochrome $\mathrm{P} 450 \mathrm{~s}$ by curcumin and curcumin decomposition products. Toxicology 235:83-91 
Asha H, Nagy I, Kovacs G, Stetson D, Ando I, Dearolf CR (2003) Analysis of Ras-induced overproliferation in Drosophila hemocytes. Genetics 163:203-215

Babcock DT, Brock AR, Fish GS, Wang Y, Perrin L, Krasnow MA, Galko MJ (2008) Circulating blood cells function as a surveillance system for damaged tissue in Drosophila larvae. Proc Natl Acad Sci USA 105:10017-10022

Becker S, Gehrsitz A, Bork P, Buchner S, Buchner E (2001) The black-pearl gene of Drosophila defines a novel conserved protein family and is required for larval growth and survival. Gene 262:15-22

Blum KS, Pabst R (2007) Lymphocyte numbers and subsets in the human blood. Do they mirror the situation in all organs? Immunol Lett 108:45-51

Brand AH, Perrimon N (1993) Targeted gene expression as a means of altering cell fates and generating dominant phenotypes. Development 118:401-415

Brennan CA, Delaney JR, Schneider DS, Anderson KV (2007) Psidin is required in Drosophila blood cells for both phagocytic degradation and immune activation of the fat body. Curr Biol 17:67-72

Bruckner K, Kockel L, Duchek P, Luque CM, Rorth P, Perrimon N (2004) The PDGF/VEGF receptor controls blood cell survival in Drosophila. Dev Cell 7:73-84

Brunner E, Peter O, Schweizer L, Basler K (1997) Pangolin encodes a Lef-1 homologue that acts downstream of Armadillo to transduce the Wingless signal in Drosophila. Nature 385:829-833

Cha GH, Cho KS, Lee JH, Kim M, Kim E, Park J, Lee SB, Chung J (2003) Discrete functions of TRAF1 and TRAF2 in Drosophila melanogaster mediated by c-Jun N-terminal kinase and NF-kappaBdependent signaling pathways. Mol Cell Biol 23:7982-7991

Charroux B, Royet J (2009) Elimination of plasmatocytes by targeted apoptosis reveals their role in multiple aspects of the Drosophila immune response. Proc Natl Acad Sci USA 106:9797-9802

Crozatier M, Meister M (2007) Drosophila haematopoiesis. Cell Microbiol 9:1117-1126

Dale E, Davis M, Faustman DL (2006) A role for transcription factor NF-kappaB in autoimmunity: possible interactions of genes, sex, and the immune response. Adv Physiol Educ 30:152-158

Davis CA, Crowley LJ, Barber MJ (2004) Cytochrome b5 reductase: the roles of the recessive congenital methemoglobinemia mutants P144L, L148P, and R159*. Arch Biochem Biophys 431:233-244

Davydov DR (2001) Microsomal monooxygenase in apoptosis: another target for cytochrome c signaling? Trends Biochem Sci 26:155-160

Duvoix A, Blasius R, Delhalle S, Schnekenburger M, Morceau F, Henry E, Dicato M, Diederich M (2005) Chemopreventive and therapeutic effects of curcumin. Cancer Lett 223:181-190

El-Lakkany NM, Seif el-Din SH, Badawy AA, Ebeid FA (2004) Effect of artemether alone and in combination with grapefruit juice on hepatic drug-metabolising enzymes and biochemical aspects in experimental Schistosoma mansoni. Int J Parasitol 34:1405-1412

Evans CJ, Banerjee U (2003) Transcriptional regulation of hematopoiesis in Drosophila. Blood Cells Mol Dis 30:223-228

Evans CJ, Hartenstein V, Banerjee U (2003) Thicker than blood: conserved mechanisms in Drosophila and vertebrate hematopoiesis. Dev Cell 5:673-690

Finn RD, McLaughlin LA, Ronseaux S, Rosewell I, Houston JB, Henderson CJ, Wolf CR (2008) Defining the in vivo role for cytochrome b5 in cytochrome P450 function through the conditional hepatic deletion of microsomal cytochrome b5. J Biol Chem 283:31385-31393

Gateff E (1994) Tumor-suppressor genes, hematopoietic malignancies and other hematopoietic disorders of Drosophila melanogaster. Ann N Y Acad Sci 712:260-279

Gilbert LI (2004) Halloween genes encode P450 enzymes that mediate steroid hormone biosynthesis in Drosophila melanogaster. Mol Cell Endocrinol 215:1-10

Giordano SJ, Kaftory A, Steggles AW (1994) A splicing mutation in the cytochrome b5 gene from a patient with congenital methemoglobinemia and pseudohermaphrodism. Hum Genet 93:568-570

Gupta A, Vij G, Sharma S, Tirkey N, Rishi P, Chopra K (2009) Curcumin, a polyphenolic antioxidant, attenuates chronic fatigue syndrome in murine water immersion stress model. Immunobiology 214:33-39

Hegesh E, Hegesh J, Kaftory A (1986) Congenital methemoglobinemia with a deficiency of cytochrome b5. N Engl J Med 314:757-761

Huppert FA, Solomou W, O'Connor S, Morgan K, Sussams P, Brayne C (1998) Aging and lymphocyte subpopulations: whole-blood analysis of immune markers in a large population sample of healthy elderly individuals. Exp Gerontol 33:593-600

Jagetia GC, Aggarwal BB (2007) "Spicing up" of the immune system by curcumin. J Clin Immunol 27:19-35 
Juvonen RO, Iwasaki M, Negishi M (1992) Roles of residues 129 and 209 in the alteration by cytochrome b5 of hydroxylase activities in mouse 2A P450S. Biochemistry 31:11519-11523

Kimbrell DA, Beutler B (2001) The evolution and genetics of innate immunity. Nat Rev Genet 2:256-267

Kimbrell DA, Hice C, Bolduc C, Kleinhesselink K, Beckingham K (2002) The Dorothy enhancer has Tinman binding sites and drives hopscotch-induced tumor formation. Genesis 34:23-28

Kornbluth S, White K (2005) Apoptosis in Drosophila: neither fish nor fowl (nor man, nor worm). J Cell Sci 118:1779-1787

Kurian JR, Longlais BJ, Trepanier LA (2007) Discovery and characterization of a cytochrome b5 variant in humans with impaired hydroxylamine reduction capacity. Pharmacogenet Genomics 17:597-603

Kwon KH, Barve A, Yu S, Huang MT, Kong AN (2007) Cancer chemoprevention by phytochemicals: potential molecular targets, biomarkers and animal models. Acta Pharmacol Sin 28:1409-1421

Lemaitre B, Hoffmann J (2007) The host defense of Drosophila melanogaster. Annu Rev Immunol 25:697-743

Li WX (2008) Canonical and non-canonical JAK-STAT signaling. Trends Cell Biol 18:545-551

Lin Z, Lou Y, Peacock J, Squires EJ (2005) A novel polymorphism in the $5^{\prime}$ untranslated region of the porcine cytochrome b5 (CYB5) gene is associated with decreased fat androstenone level. Mamm Genome 16:367-373

Locuson CW, Wienkers LC, Jones JP, Tracy TS (2007) CYP2C9 protein interactions with cytochrome b(5): effects on the coupling of catalysis. Drug Metab Dispos 35:1174-1181

Luo H, Hanratty WP, Dearolf CR (1995) An amino acid substitution in the Drosophila hopTum-1 Jak kinase causes leukemia-like hematopoietic defects. EMBO J 14:1412-1420

Mandal L, Martinez-Agosto JA, Evans CJ, Hartenstein V, Banerjee U (2007) A Hedgehog- and Antennapedia-dependent niche maintains Drosophila haematopoietic precursors. Nature 446:320-324

Markus R, Laurinyecz B, Kurucz E, Honti V, Bajusz I, Sipos B, Somogyi K, Kronhamn J, Hultmark D, Ando I (2009) Sessile hemocytes as a hematopoietic compartment in Drosophila melanogaster. Proc Natl Acad Sci USA 106:4805-4809

Marriott I, Huet-Hudson YM (2006) Sexual dimorphism in innate immune responses to infectious organisms. Immunol Res 34:177-192

Means JC, Hays R (2007) Mitochondrial membrane depolarization in Drosophila apoptosis. Cell Death Differ 14:383-385

Meister M, Lagueux M (2003) Drosophila blood cells. Cell Microbiol 5:573-580

Miller WL (2005) Minireview: regulation of steroidogenesis by electron transfer. Endocrinology 146:2544-2550

Minakhina S, Steward R (2006a) Nuclear factor-kappa B pathways in Drosophila. Oncogene 25:6749-6757

Minakhina S, Steward R (2006b) Melanotic mutants in Drosophila: pathways and phenotypes. Genetics 174:253-263

Oberst A, Bender C, Green DR (2008) Living with death: the evolution of the mitochondrial pathway of apoptosis in animals. Cell Death Differ 15:1139-1146

Ow YP, Green DR, Hao Z, Mak TW (2008) Cytochrome c: functions beyond respiration. Nat Rev Mol Cell Biol 9:532-542

Pili-Floury S, Leulier F, Takahashi K, Saigo K, Samain E, Ueda R, Lemaitre B (2004) In vivo RNA interference analysis reveals an unexpected role for GNBP1 in the defense against Gram-positive bacterial infection in Drosophila adults. J Biol Chem 279:12848-12853

Portoles MT, Diaz-Laviada I, Ainaga MJ, Pagani R, Municio AM (1989) Involvement of cytochrome b5 in the cytotoxic response to Escherichia coli lipopolysaccharide. Mol Cell Biochem 87:79-84

Reichert T, DeBruyere M, Deneys V, Totterman T, Lydyard P, Yuksel F, Chapel H, Jewell D, Van Hove L, Linden J (1991) Lymphocyte subset reference ranges in adult Caucasians. Clin Immunol Immunopathol 60:190-208

Remillieux-Leschelle N, Santamaria P, Randsholt NB (2002) Regulation of larval hematopoiesis in Drosophila melanogaster: a role for the multi sex combs gene. Genetics 162:1259-1274

Rizki TM, Rizki RM (1983) Blood cell surface changes in Drosophila mutants with melanotic tumors. Science 220:73-75

Rodriguez A, Zhou Z, Tang ML, Meller S, Chen J, Bellen H, Kimbrell DA (1996) Identification of immune system and response genes, and novel mutations causing melanotic tumor formation in Drosophila melanogaster. Genetics 143:929-940

Sa G, Das T (2008) Anti cancer effects of curcumin: cycle of life and death. Cell Div 3:14 
Schenkman JB, Jansson I (1999) Interactions between cytochrome P450 and cytochrome b5. Drug Metab Rev 31:351-364

Schenkman JB, Jansson I (2003) The many roles of cytochrome b5. Pharmacol Ther 97:139-152

Shames RS (2002) Gender differences in the development and function of the immune system. J Adolesc Health 30:59-70

Sheweita SA (2004) Carcinogen-metabolizing enzymes and insecticides. J Environ Sci Health B 39:805-818

Shia AK, Glittenberg M, Thompson G, Weber AN, Reichhart JM, Ligoxygakis P (2009) Toll-dependent antimicrobial responses in Drosophila larval fat body require Spatzle secreted by haemocytes. J Cell Sci 122:4505-4515

Sinenko SA, Kim EK, Wynn R, Manfruelli P, Ando I, Wharton KA, Perrimon N, Mathey-Prevot B (2004) Yantar, a conserved arginine-rich protein is involved in Drosophila hemocyte development. Dev Biol 273:48-62

Spradling AC, Stern D, Beaton A, Rhem EJ, Laverty T, Mozden N, Misra S, Rubin GM (1999) The Berkeley Drosophila genome project gene disruption project: single P-element insertions mutating 25\% of vital Drosophila genes. Genetics 153:135-177

Stofanko M, Kwon SY, Badenhorst P (2008) A misexpression screen to identify regulators of Drosophila larval hemocyte development. Genetics 180:253-267

Taylor K, Kimbrell DA (2007) Host immune response and differential survival of the sexes in Drosophila. Fly (Austin) 1:197-204

Tepass U, Fessler LI, Aziz A, Hartenstein V (1994) Embryonic origin of hemocytes and their relationship to cell death in Drosophila. Development 120:1829-1837

Tirouvanziam R, Davidson CJ, Lipsick JS, Herzenberg LA (2004) Fluorescence-activated cell sorting (FACS) of Drosophila hemocytes reveals important functional similarities to mammalian leukocytes. Proc Natl Acad Sci USA 101:2912-2917

Tweedie S, Flybase Consortium (2009) FlyBase: enhancing Drosophila gene ontology annotations. Nucleic Acids Res 37:D555-D559

Umbreit J (2007) Methemoglobin-it's not just blue: a concise review. Am J Hematol 82:134-144

Vass E, Nappi AJ (2000) Developmental and immunological aspects of Drosophila-parasitoid relationships. J Parasitol 86:1259-1270

Wang L, Ligoxygakis P (2006) Pathogen recognition and signalling in the Drosophila innate immune response. Immunobiology 211:251-261

Wang L, Cowley AB, Terzyan S, Zhang X, Benson DR (2007) Comparison of cytochromes b5 from insects and vertebrates. Proteins 67:293-304

Watson KL, Johnson TK, Denell RE (1991) Lethal(1) aberrant immune response mutations leading to melanotic tumor formation in Drosophila melanogaster. Dev Genet 12:173-187

Watson KL, Konrad KD, Woods DF, Bryant PJ (1992) Drosophila homolog of the human S6 ribosomal protein is required for tumor suppression in the hematopoietic system. Proc Natl Acad Sci USA 89:11302-11306

Wilson C, Pearson RK, Bellen HJ, O’Kane CJ, Grossniklaus U, Gehring WJ (1989) P-element-mediated enhancer detection: an efficient method for isolating and characterizing developmentally regulated genes in Drosophila. Genes Dev 3:1301-1313

Wingender E (2008) The Transfac project as an example of framework technology that supports the analysis of genomic regulation. Brief Bioinform 9:326-332

Wu H, Arron JR (2003) TRAF6, a molecular bridge spanning adaptive immunity, innate immunity and osteoimmunology. Bioessays 25:1096-1105

Yamaori S, Yamazaki H, Suzuki A, Yamada A, Tani H, Kamidate T, Fujita K, Kamataki T (2003) Effects of cytochrome b(5) on drug oxidation activities of human cytochrome P450 (CYP) 3As: similarity of CYP3A5 with CYP3A4 but not CYP3A7. Biochem Pharmacol 66:2333-2340

Yamazaki H, Shimada T (2006) Cytochrome P450 reconstitution systems. Methods Mol Biol 320: 61-71

Yamazaki H, Nakamura M, Komatsu T, Ohyama K, Hatanaka N, Asahi S, Shimada N, Guengerich FP, Shimada T, Nakajima M, Yokoi T (2002) Roles of NADPH-P450 reductase and apo- and holocytochrome b5 on xenobiotic oxidations catalyzed by 12 recombinant human cytochrome P450s expressed in membranes of Escherichia coli. Protein Expr Purif 24:329-337

Ye YH, Chenoweth SF, McGraw EA (2009) Effective but costly, evolved mechanisms of defense against a virulent opportunistic pathogen in Drosophila melanogaster. PLoS Pathog 5:e1000385 
Zandman-Goddard G, Peeva E, Shoenfeld Y (2007) Gender and autoimmunity. Autoimmun Rev 6:366-372

Zettervall CJ, Anderl I, Williams MJ, Palmer R, Kurucz E, Ando I, Hultmark D (2004) A directed screen for genes involved in Drosophila blood cell activation. Proc Natl Acad Sci USA 101:14192-14197 